\title{
A contabilidade do carbon footprint: uma revisão integrativa
}

O objetivo deste artigo é identificar evidências disponíveis na literatura sobre às práticas da contabilidade em relação à Pegada de Carbono. Para isso foi utilizada a Revisão integrativa de artigos publicados em inglês, disponíveis na íntegra nas bases Scorpus e Web of Science. Foram utilizadas as palavras chaves 'contabilidade de carbono', 'carbon accounting', 'pegada de carbono' e 'carbon footprint', respectivamente, sendo selecionados e analisados dezesseis estudos. Após as etapas da revisão integrativa de literatura restaram 16 artigos que foram selecionados para a análise inicial do tema. Como resultado, foi possível identificar que a definição de contabilidade de emissões de carbono envolve o processo de mitigação como um caminho mais acessível para que as organizações possam lidar com a pegada de carbono. Apesar de quantificar as emissões de maneira global, a forma que os resultados são obtidos são variáveis pelo cálculo e foco da medição. Deste modo, os tomadores de decisão das organizações precisam considerar os diversos regulamentos legais às emissões de carbono.

Palavras-chave: Pegada de Carbono; Contabilidade de Carbono; Custos; ODS.

\section{Carbon footprint accounting: an integrative review}

The purpose of this article is to identify evidence available in the literature on accounting practices in relation to the Carbon Footprint. For this purpose, an integrative review of articles published in English was used, available in full on the Scorpus and Web of Science databases. The keywords 'carbon accounting', 'carbon accounting', 'carbon footprint' and 'carbon footprint' were used, respectively, and sixteen studies were selected and analyzed. After the steps of the integrative literature review, 16 articles remained, which were selected for the initial analysis of the topic. As a result, it was possible to identify that the definition of carbon emissions accounting involves the mitigation process as a more accessible way for organizations to deal with the carbon footprint. Despite quantifying emissions globally, the way the results are obtained is variable by the calculation and focus of the measurement. Therefore, organizations' decision makers need to consider the various legal regulations on carbon emissions.

Keywords: Carbon Footprint; Carbon Accounting; Costs; SDGs.

Topic: Planejamento, Gestão e Políticas Públicas Ambientais

Reviewed anonymously in the process of blind peer.
Received: 04/06/2021

Approved: 29/06/2021
Alexsandro da Silva (D)

Universidade do Sul de Santa Catarina, Brasil http://lattes.cnpq.br/9142330909980362 http://orcid.org/0000-0002-5316-7563

alexsandrodasilva3@gmail.com

\section{Stela Bueno Mergner}

Universidade do Sul de Santa Catarina, Brasil

http://lattes.cnpq.br/5320702802789430

stela.bueno@icloud.com

Ana Regina de Aguiar Dutra (iD)

Universidade do Sul de Santa Catarina, Brasil

http://lattes.cnpq.br/3147098935379439

http://orcid.org/0000-0002-0337-5639

aradutra@gmail.com

\section{Sandro Vieira Soares (iD) \\ Universidade do Sul de Santa Catarina, Brasil \\ http://lattes.cnpq.br/0428315375726151 \\ http://orcid.org/0000-0001-7076-4936 \\ sandrovieirasoares@hotmail.com}

Referencing this:

SILVA, A.; MERGNER, S. B.; DUTRA, A. R. A.; SOARES, S. V.. A contabilidade do carbon footprint: uma revisão integrativa. Revista Ibero Americana de Ciências Ambientais, v.12, n.6, p.625-634, 2021. DOI: http://doi.org/10.6008/CBPC2179-6858.2021.006.0052 


\section{INTRODUÇÃO}

A Política Nacional sobre Mudança do Clima, instituída pela Lei no 12.187, de 29 de dezembro de 2009, conceitua os Gases de Efeito Estufa como constituintes gasosos, naturais ou emitidos por fontes antrópicas, que, na atmosfera, absorvem e reemitem radiação infravermelha.

Vale destacar que os Gases de Efeito Estufa são fundamentais para a ocorrência do Efeito Estufa, o qual é um fenômeno natural e benéfico, cujos alguns tipos de gases agem como uma estufa e dificultam o retorno da radiação solar para o espaço, mantendo a temperatura da terra num nível adequado para o desenvolvimento e manutenção da vida no planeta (SCHWARTZ, 2018).

Neste contexto, destaque-se a necessidade de executar a Contabilidade de Carbono (Carbon Accounting), que compreende a necessidade de conhecer, avaliar monetariamente ou não e monitorar as emissões de Gases de Efeito Estufa em todos os níveis da cadeia de valores, além de conhecer, avaliar e monitorar os efeitos dessas emissões no ciclo de carbono dos ecossistemas (STECHEMESSER et al., 2012). Conforme estes autores, o Carbon Accounting pode ser de execução obrigatória ou voluntária e em diferentes escalas, tais como: global, nacional, regional, local e organizacional. Nele, estão abarcados conceitos e metodologias para quantificação da Pegada de Carbono, que extrapolando o conceito de Wiedmann e Minx é entendida como a contabilização dos Gases de Efeito Estufa que, direta e indiretamente, são emitidos por uma atividade ou produto, incluindo bens e serviços, de um indivíduo, população, empresa, instituições públicas, cidade, estado, país.

A Pegada de Carbono também é compreendida como um indicador ambiental que possibilita orientar decisões sobre consumo e direcionar o planejamento, execução e o monitoramento da eficiência das políticas e ações de redução e controle das emissões de Gases de Efeito Estufa (ALVAREZ et al., 2016; ALVAREZ et al., 2014; STECHEMESSER et al., 2012). No entanto, diferenças entre as abordagens para a Pegada de Carbono Organizacional e de Pegada de Carbono de Produto podem prejudicar a aplicação mais ampla da Pegada de Carbono (ALVAREZ et al., 2014).

Metodologias para o cálculo da Pegada de Carbono Organizacional foram estabelecidas baseadas nas diretrizes para a elaboração dos inventários nacionais do Painel Intergovernamental sobre Mudanças Climáticas (Intergovernmental Panel on Climate Change - IPCC), de 1996 e 2006, a exemplo do padrão internacional ISO 14064-1 e GHG Protocol (ALVAREZ et al., 2014).

Assim a presente pesquisa pretende responder a seguinte indagação: quais métodos de custos estão sendo empregados para tratar a pegada de carbono?. Para responder a esta pergunta, o presente trabalho tem como objetivo geral analisar e descrever as características das publicações sobre o tema Contabilidade de Carbono e a Pegada de Carbono.

\section{REVISÃO TEÓRICA}

\section{Pegada de carbono}

Como visto, nos últimos anos houve um crescimento constante do número de empresas 
comprometidas com ações climáticas, em função do aprofundamento científico sobre o aquecimento global, do atual Acordo de Paris, das estratégias empresariais e mercadológicas e das pressões legais e socioambientais (ALVAREZ et al., 2016).

Com isso, diversas organizações passaram a reconhecer suas Pegadas de Carbono, cuja ideia deriva da Pegada Ecológica, que por sua vez mensura a área de terra necessária para a produção e manutenção de bens e serviços consumidos, incluindo alimentos, água, energia e eliminação de resíduos, por pessoa, produto, serviço, cidade, país, etc. (HRASKY, 2011).

Portanto, a Pegada de Carbono é entendida como a contabilização dos Gases de Efeito Estufa que, direta e indiretamente, é emitido por uma atividade, processo ou produto, incluindo bens e serviços, de um indivíduo, população, empresa, instituições públicas, cidade, Estado e/ou País (ZVEZDOV et al., 2015). Ascui et al. (2011) corrobora ao afirmar que a Pegada de Carbono de uma unidade funcional (organização, cidade, produto, serviço, processo etc.) é o impacto climático, sob uma métrica especificada, considerando todas as fontes de emissão tanto na produção, quanto no consumo.

Embora ainda não haja um conceito padrão, já é pacífico que a Pegada de Carbono é um indicador ambiental que possibilita a tomada de decisões de forma ambientalmente mais adequada, com capacidade de direcionar o planejamento, execução e monitoramento da eficiência de políticas e ações para a redução e controle das emissões de Gases de Efeito Estufa (ALVAREZ et al., 2016; ALVAREZ et al., 2014).

A medição da Pegada de Carbono é um novo desafio, em especial, para organizações que tentam adotar uma gestão sustentável, reduzir seus custos por meio da eficiência ecológica, bem como desenvolver e aplicar estratégias de gestão das emissões de Gases de Efeito Estufa. Consoante com Alvarez et al. (2014), a Pegada de Carbono pode ser analisada para diversas unidades funcionais, considerando diferentes escalas e métodos.

\section{Contabilidade de carbono}

Diante dos desafios impostos pelas alterações do clima, contabilizar as emissões de Gases de Efeito Estufa se torna cada vez mais necessária para as organizações a fim de subsidiar seus processos decisórios, num cenário em que as ações para mitigação e controle das emissões de Gases de Efeito Estufa têm sido exigidas ou incentivadas pelos clientes, acionistas, sociedade e legislação. Assim sendo, diversas organizações, de forma voluntária ou não, estão desenvolvendo a capacidade para medir, monitorar e relatar suas emissões de Gases de Efeito Estufa, por meio do Carbon Accounting ou Contabilidade do Carbono (SCHALTEGGER et al., 2012; STECHEMESSER et al., 2012).

Schaltegger et al. (2012) destacam que a Contabilidade de Carbono encontra amparo nas ações de Responsabilidade Social Corporativa e, notadamente, no paradigma do Desenvolvimento Sustentável, o qual busca um modelo de crescimento econômico e social aliado à preservação e conservação ambiental e ao equilíbrio climático no planeta, aliado a alcançar os ODS (Objetivos de Desenvolvimento Sustentável).

Ainda não há uma definição única para Contabilidade de Carbono, este termo pode significar diferentes coisas para diferentes pessoas, podendo ser entendido como a atividade de mensurar as emissões 
e remoções de Gases de Efeito Estufa e a manutenção de um inventário permanente de emissões, ou se referir aos impactos nas demonstrações financeiras resultantes do ambiente regulatório de carbono de uma entidade, ou ainda, estar relacionado à medição e comercialização de emissões de carbono (ASCUI et al., 2011; STECHEMESSER et al., 2012).

A Contabilidade de Carbono compreende o cálculo, a avaliação monetária e não monetária, o monitoramento das emissões de Gases de Efeito Estufa em todos os níveis da cadeia de valor, bem como o cálculo, avaliação e o monitoramento dos efeitos dessas emissões no ciclo de carbono dos ecossistemas (STECHEMESSER et al., 2012). Na escala Organizacional possui dimensão física e não física, a primeira engloba a Pegada de Carbono (Carbon Footprint) e, a segunda, abarca questões monetárias e estratégicas para a organização, enfocando a contabilidade gerencial ou contabilidade de custos (ASCUI et al., 2011).

Assim, a Contabilidade de Carbono oferece suporte para que uma organização execute sua gestão de carbono, por meio da identificação, coleta, processamento e divulgação de informações atinentes às emissões de carbono. Neste contexto, considerando os objetivos da pesquisa, será dado enfoque na Contabilidade de Carbono Organizacional, mais especificamente na Pegada de Carbono (ASCUI et al., 2011).

\section{METODOLOGIA}

A metodologia foi delineada e executada para atingir seus objetivos e resultados finais, sendo subdivididas em: (i) enquadramento metodológico, (ii) instrumento de intervenção e (iii) processo de análise e de tratamento de dados.

\section{Enquadramento metodológico}

A presente pesquisa é de natureza exploratório-descritiva (RICHARDSON, 2017). É exploratória por proporcionar uma investigação sobre a Contabilidade de Carbono e a Pegada de Carbono, permitindo uma visão mais ampla sobre o assunto e, descritiva, por permitir estudar as características de um determinado grupo de variáveis (RICHARDSON, 2017).

No que tange à natureza da pesquisa, é denominada como teórico-ilustrativa. Tendo em vista que, de acordo com Ferreira et al. (2004) tem o objetivo de ilustrar a operacionalização do instrumento, para busca e análise bibliométrica de artigos sobre o tema da avaliação da produção científica de pesquisadores, a fim de que outros pesquisadores possam fazer uso desse instrumento e, assim, conseguir selecionar um portfólio bibliográfico relevante e alinhado ao tema de pesquisa que estiverem investigando.

A lógica da pesquisa é indutiva por realizar a observação de informações de um problema cujo conhecimento se pretende adquirir e explicitá-lo durante o decorrer do trabalho (GIL, 2008).

Os dados foram coletados de forma primária, originários das delimitações postas pelos pesquisadores para o processo de seleção das publicações a fim de compor o portfólio bibliográfico por meio de abordagem qualitativa; e de forma secundária, quando os artigos selecionados, assim como as informações relacionadas, foram extraídos de fonte comuns à comunidade científica (RICHARDSON, 2017).

Os resultados da pesquisa se caracterizam como aplicada, permitindo que o conhecimento adquirido 
seja utilizado para a realização dos objetivos específicos (RICHARDSON, 2017). Adotou-se a pesquisa bibliográfica como procedimento técnico pelo fato de compor um arcabouço teórico (GIL, 2008).

\section{Instrumento de Intervenção}

Para analisar como os periódicos das áreas de Administração e Contabilidade estão abordando o tema 'pegada de carbono' e 'contabilidade de carbono', foi empreendida uma revisão integrativa da literatura. Esse tipo de estudo busca identificar publicações sobre uma temática com o objetivo de recolher informações ou conhecimentos prévios sobre o problema a respeito do qual se procura a resposta (FONSECA, 2002). Visando dar uma melhor contribuição sobre o tema para a academia, também foram analisados os resultados encontrados em cada artigo, uma análise que pode ser defina por ser uma revisão integrativa.

A revisão é uma maneira apropriada de resolver o problema e a integração e síntese de uma revisão da literatura pode fornecer a base para desenvolver a teoria através de pesquisas futuras. Carey (2012) ressalta que a revisão integrativa da literatura é uma forma distinta de pesquisa que gera novos conhecimentos sobre o tema revisado. Como proposto no início deste artigo, a questão de pesquisa a ser respondida nesse trabalho diz respeito aos métodos de custos para tratarem da pegada de carbono. É desta questão que nascem as estratégias de busca para se obter uma gama de evidências científicas.

\section{Seleção do Portfólio Bibliográfico}

Nessa pesquisa, as bases de dados empregadas foram Scopus e Web of Science, com as estratégias de busca 'carbono footprint' AND 'carbon accounting', dentro do limite que compreendeu os anos de 2010 a 2020, a fim de abranger o maior quantitativo de publicações. Na base de dados Scopus foram selecionados 92 resultados e na Web of Science 41 resultados. Após o processo de seleção e identificação dos artigos que obedeceram aos seguintes critérios, prévia leitura de todos os títulos e resumos, selecionou-se 51 artigos, que tiveram seu resumo lido para verificar se estavam enquadrados no tema 'carbono footprint' and 'carbon accounting'. Ao final desta etapa, 4 artigos foram retirados da análise por não abordarem o assunto. Dos 47 artigos restantes, foram selecionadas apenas as publicações em periódicos de alto impacto, visando obter uma melhor qualidade dos artigos. Restaram 16 artigos que foram selecionados para essa análise inicial do tema.

\section{Processo de Análise e tratamento de Dados}

Tabela 1: Relevância de revistas pesquisadas com os temas da pesquisa.

\begin{tabular}{l|l}
\hline Relevância & Quantidade de Artigos \\
\hline Resultado da pesquisa na base de dados na Scopus e Web of Science & $\mathbf{1 3 3}$ \\
\hline Relevância com o tema Contabilidade de Carbono & $\mathbf{1 6}$ \\
\hline Irrelevância com o tema Contabilidade de Carbono & $\mathbf{4}$ \\
\hline
\end{tabular}

A partir da execução da primeira e segunda etapas foi efetuado o mapeamento dos artigos da pesquisa de forma abrangente. Além disso, os pesquisadores classificaram os artigos em duas categorias, a saber, periódicos relevantes ao tema Contabilidade de carbono e pegado de carbono e periódicos 
irrelevantes conforme destaca a tabela 1. Em resumo, confirma-se que dos artigos selecionados 16 são relevantes para a sistematização dos conhecimentos.

\section{Etapa 3 - Avaliação da Qualidade}

Após a classificação de acordo com a tabela 1, os pesquisadores realizaram outros agrupamentos para os artigos que foram considerados relevantes para os temas pesquisados. Os 16 artigos foram agrupados em quatro grupos principais, em função dos conteúdos de discussão, conforme tabela 2: 1) artigos que discutem a definição de pegada de carbono; 2) artigos que abordam a definição de contabilidade de carbono; 3) artigos que debatem a aplicação da contabilidade de carbono; 4) artigos que discutem a avaliação da contabilidade do carbono.

Tabela 2: Grupo de artigos com foco de pesquisa.

\begin{tabular}{l|l}
\hline Foco de Pesquisa & Quantidade de Artigos \\
\hline Definição de Pegada de carbono & 2 artigos \\
\hline Definição de contabilidade de carbono & 4 artigos \\
\hline Aplicação da contabilidade de carbono & 5 artigos \\
\hline Avaliação da contabilidade de carbono & 5 artigos \\
\hline Total & $\mathbf{1 6}$ Revistas \\
\hline
\end{tabular}

Os agrupamentos dos artigos relevantes consistem em dois artigos que discutem a definição de pegada de carbono periódicos, quatro artigos que discutem a definição de contabilidade de carbono, cinco artigos que discutem a aplicação da contabilidade de carbono e cinco artigos que discutem a avaliação da contabilidade do carbono.

A tabela 2 nos mostra o resultado dos agrupamentos, onde 2 artigos trazem a definição de pegada de carbono periódicos, 4 discutem a definição de contabilidade de carbono, 5 artigos debatem a aplicação da contabilidade de carbono e, ainda, 5 artigos discutem a avaliação da contabilidade do carbono.

\section{Etapa 4 - extração de dados}

O processo de extração dos dados foi realizado pela leitura completa dos artigos selecionados. Com base nos resultados da extração dos dados, foi possível identificar que a Definição de Pegada de carbono se encontravaem 2 artigos publicados nos periódicos Journal of Cleaner Production e Accounting, Auditing and Accountability Journal. Quanto à definição acerca da Contabilidade de Carbono, esta foi encontrada em 3 artigos no periódico Journal of Cleaner Production e 1 artigo no periódico Corporate Carbon and Climate Accounting.

No tocante da discussão acerca da Aplicação da Contabilidade de Carbono, encontrou-se 5 artigos, 1 em cada periódico a seguir: Accounting, Auditing and Accountability Journal; Science of the total Environment; Antipode, Social Responsibility Journal; Pacific Accounting Review. Já em relação à Avaliação de Pegada de Carbono, apresentaram 2 artigos cada, enquanto o periódico Energy Economics apresentou 1 artigo. 


\section{RESULTADO}

\section{Definição de contabilidade de carbono}

Foram identificados dois artigos que explicam detidamente a definição de contabilidade de carbono, que são das autorias de Ascui et al. (2011) e Zvezdov et al. (2015). Ascui et al. (2011) dividem conceito de contabilidade de carbono em cinco estruturas principais: contabilidade física do carbono, contabilidade política de carbono, carbono habilitador do mercado contabilidade, contabilidade financeira de carbono e carbono social/contabilidade ambiental.

Além disso, Zvezdov et al. (2015) conduziram mais pesquisas sobre a aplicação da contabilidade do carbono através da contabilidade gerencial do carbono. Eles definem a contabilidade da gestão de carbono como uma atividade relacionada ao objetivo de alcançar o uso eficiente de recursos, considerando o tempo de uso dos recursos, e redução efetiva do carbono com a motivação de legitimidade orientada para lucro e custo. Assim, pode-se concluir que a definição de contabilidade de carbono é um processo de medição da emissão de carbono produzida por uma organização, fornecendo esforços para reduzir e relatar resultados para as partes interessadas, a fim de apoiar o desenvolvimento sustentável de suas metas.

A discussão apresentada nesses dois artigos apoia a medição das mudanças climáticas no aspecto de mitigação ao invés de adaptação. Isso está em linha com as descobertas de Stechemesser et al. (2012). A abordagem de mitigação tende a minimizar os custos do efeito global do aquecimento por meio de metas de redução.

\section{Aplicação da contabilidade de carbono}

Foram encontrados cinco artigos que examinam a aplicação contábil do carbono em vários aspectos. O primeiro estudo foi conduzido por Hrasky (2011), que discute a organização relatando a divulgação da pegada de carbono (carbon footprint), para estabelecer um debate entre atores da sociedade sobre a divulgação acerca da pegada de carbono. As organizações querem legitimidade para agir com base em valores aceitáveis em termos da pegada de carbono. Este fato descoberto por Hrasky (2011) tornou-se uma questão interessante para uma investigação mais aprofundada, devido a um debate entre elementos da sociedade em relação à divulgação de carbono.

A aplicação de uma contabilidade de gestão de carbono eficaz pode fornecer benefícios financeiros para organizações, especialmente aquelas envolvidas em setores intensivos em carbono, como benefícios fiscais sobre o carbono (JAMALUDIN et al., 2019). Em 2015, Caro et. al. (2015) já haviam mostrado que organizações que implementam a medição do carbono são mais beneficiadas em termos financeiros.

As organizações profissionais de contabilidade têm um papel importante na implementação da contabilidade de carbono, uma vez que os contadores veem as mudanças climáticas como um problema sério para a sustentabilidade (LOVELL et al., 2012).

Melo (2012) constatou que o desempenho social corporativo era diretamente proporcional à cultura organizacional e à gestão. Choi et al. (2013) corroboram com Melo (2012) ao salientarem que o desempenho 
organizacional é composto por vários aspectos, um deles é a contabilidade de carbono. É importante destacar o impacto positivo obtido pela organização quando esta presta conta a todos os seus stakeholders, incluindo a sociedade, das suas ações em termos de pegada de carbono.

As organizações que fazem relatórios voluntários de emissões de gases de efeito estufa e aplicam um sistema de gestão ambiental, têm um bom sistema de governança organizacional e conduzem relatórios abertos sobre o Carbon Disclosure Project (uma das organizações sem fins lucrativos/ONGs, que incentivam o relato das emissões de carbono nas organizações (RANKIN et al., 2011). A implementação da divulgação de emissões de carbono é influenciada por vários fatores, um dos quais é a boa governança. Isso funcionará de forma eficaz se a empresa tiver uma boa gestão ambiental (CHOI et al., 2013; RANKIN et al., 2011).

\section{Medição da contabilidade de carbono}

Em relação à medição da contabilidade de carbono, foram identificados em quatro periódicos relevantes relacionados ao tópico principal. Um autor menciona várias referências para medição da contabilidade do carbono. Essas medições de emissão de carbono os métodos são mostrados na tabela 3.

Tabela 3: Referências para medição de Contabilidade de Carbono.

\begin{tabular}{|c|c|c|}
\hline Referência & Intensidade de medição & Base para Cálculo \\
\hline Protocolo de Kyoto & $\begin{array}{l}\text { - A intensidade da medição não é explicada } \\
\text { - O cálculo da emissão é uma função de dados de atividade, envolvendo } \\
\text { observações diretas às emissões ou aplicações do fator de emissão }\end{array}$ & Atividade de produção \\
\hline $\begin{array}{l}\text { Diretivas } \quad \text { CE } \\
\text { 2007/589; } \\
\text { 2003/87 Anexo IV; } \\
96 / 61\end{array}$ & $\begin{array}{l}\text { - A intensidade da medição não é explicada } \\
\text { - O cálculo da emissão é uma função de dados de atividade, fatores de } \\
\text { emissão e fatores de oxidação }\end{array}$ & $\begin{array}{l}\text { Depende do acordado } \\
\text { ou métodos } \\
\text { padronizados }\end{array}$ \\
\hline $\begin{array}{l}\text { Análise de Entrada- } \\
\text { Saída }\end{array}$ & $\begin{array}{l}\text { - A intensidade da medição é uma função do total de recursos consumidos, } \\
\text { custo dos recursos consumindo, dados de atividade e fator de emissão } \\
\text { - Necessidade de modelagem estrutural }\end{array}$ & $\begin{array}{l}\text { - Produção, atividade e } \\
\text { custo dados } \\
\text { - Adequado para análise } \\
\text { limitado dos setores }\end{array}$ \\
\hline $\begin{array}{l}\text { Decomposição da } \\
\text { Análise Estrutural }\end{array}$ & $\begin{array}{l}\text { A emissão é uma função da energia intensidade de emissão, que depende } \\
\text { nas taxas de atividade (por exemplo, PIB, energia usado, população, volume } \\
\text { de produção, e receita operacional de uma organização) }\end{array}$ & $\begin{array}{l}\text { Taxas de várias } \\
\text { atividades }\end{array}$ \\
\hline $\begin{array}{l}\text { Padrão British } \\
\text { Standard } 2050\end{array}$ & $\begin{array}{l}\text { - A pegada de carbono é uma função de dados e atividade e fator de emissão } \\
\text { - Os dados de atividade são considerados brutos materiais, enquanto a } \\
\text { energia usada é relacionada ao ciclo de vida do produto } \\
\text { - A incerteza dá a necessidade de calcular densidade de probabilidade } \\
\text { - Não há necessidade de relatar a emissão no cálculo }\end{array}$ & Ativic \\
\hline $\begin{array}{l}\text { Protocolo Padrões } \\
\text { GHG }\end{array}$ & $\begin{array}{l}\text { Os volumes de emissão se relacionam diretamente com organizações e } \\
\text { dependem da quantidade de produção }\end{array}$ & $\begin{array}{l}\text { Cotação do Fluxo de } \\
\text { produção }\end{array}$ \\
\hline
\end{tabular}

Embora oriundos de setores distintos, esses seis métodos compartilham o objetivo comum de quantificar as emissões de carbono, a fim de melhorar a tomada de decisões. Os cálculos das emissões pelas organizações terão um impacto positivo para a sustentabilidade organizacional e o desenvolvimento sustentável.

Além disso, Liddle (2018) compara a medição da emissão de carbono, ou seja, emissões de carbono baseadas no consumo e emissões de carbono baseadas no território. As emissões de carbono baseadas no consumo são medidas pelo cálculo do consumo doméstico de combustível, adicionado às importações líquidas (importações menos exportações). 
Ambos os métodos destacados por Liddle (2018) podem ser usados como medidas quantitativas na aplicação da contabilidade de carbono. No entanto, o alcance desses dois métodos depende das condições macroeconômicas, já que as medições usam taxas de consumo e produção em um país. Portanto, os métodos podem não ser úteis em ambientes micro ou organizacionais.

Jamaludin et al. (2019) combinam dois métodos de medidas para pegada de carbono, por exemplo, contabilidade da pegada de carbono e índice de sustentabilidade do palmito. Este autor desenvolve uma medição de sustentabilidade integrada para a indústria de óleo de palma, visando reduzir dados excessivos, introduzindo um método mais abrangente. Pesquisas comparativas relacionadas à medição de emissões de carbono estão crescendo em número e em muitas regiões (MAALOUF et al., 2018), resultando na alta variabilidade dos métodos de medição.

Apesar de compartilhar o mesmo objetivo de quantificar as emissões, as diferenças permanecem existindo devido a variações nos elementos incluídos no cálculo e o foco de cada medição (MAALOUF et al., 2018). Estes autores recomendam, ainda que uma organização internacional precisa ser estabelecida como um órgão regulador em contabilidade de carbono. As aplicações de contabilidade de carbono utilizadas por organizações tendem muitas vezes, a categorizar as ações de gestão com motivação para ganhar legitimidade ou como forma de responsabilidade para partes interessadas (STECHEMESSER et al., 2012).

Stechemesser et al. (2012), em sua revisão de artigos sobre a medição de emissões de carbono, concluíram que a medição de carbono emprega a base de produção. Isto é porque base de consumo ou abordagem baseada no território, basicamente, usa o relatório nacional de produção, o mesmo que serve como base do Protocolo de Kyoto.

\section{CONCLUSÕES}

Este artigo foi desenvolvido com o objetivo de analisar e descrever as características das publicações sobre o tema Contabilidade de Carbono e a Pegada de Carbono contabilidade de carbono, bem como a forma de aplicar e medir a contabilidade do carbono por meio de revisão integrativa da literatura. A pesquisa bibliográfica foi realizada nas bases de dados Scopus e Web of Science para os períodos de observação de 2010-2020.

As etapas da revisão integrativa de literatura seguiram o processo de seleção e identificação dos artigos, que obedeceram aos critérios de inclusão estabelecidos, prévia leitura de todos os títulos, resumos ou abstract, totalizando 51 artigos selecionados, com a estratégia de busca 'carbono footprint' AND 'carbon accounting'. Ao final desta etapa, 4 artigos foram retirados da análise por não abordarem o assunto. Dos 47 artigos restantes, foram selecionadas apenas as publicações em periódicos de alto impacto visando obter uma melhor qualidade dos artigos. Restaram 16 artigos que foram selecionados para a análise inicial do tema.

Como resultado, foi possível identificar que a definição de contabilidade de emissões de carbono trata do processo de mitigação em vez de adaptação, como um caminho mais acessível para que as organizações possam lidar com a pegada de carbono. Também, as aplicações de divulgação de emissões de carbono tendem a priorizar as necessidades das partes interessadas, o que vai ao encontro da teoria das 
partes interessadas. Para a medição das emissões de carbono é utilizada a base de produção, diferente da base de consumo ou base no território, que emprega basicamente o relatório de produção nacional, pelo Protocolo de Kyoto, por exemplo.

As limitações desta revisão sistemática referem-se ao número pequeno de artigos que abordam o tema, e muitos deles discutem quanto a natureza das divulgações das emissões carbono, categorizando as ações de gestão com motivação para ganhar legitimidade ou como forma de responsabilidade para partes interessadas, necessitando uma pesquisa mais aprofundada sobre o tema.

\section{REFERÊNCIAS}

ALVAREZ, S.; CARBALLO-PENELA, A.; MATEO-MANTECÓN, I.; RUBIO, A.. Strengths-Weaknesses-Opportunities-Threats analysis of carbon footprint indicator and derived recommendations. Journal of Cleaner Production, v.121, p.238-247, 2016. DOI: http://doi.org/10.1016/j.jclepro.2016.02.028

ALVAREZ, S.; BLANQUER, M.; RUBIO, A.. Carbon footprint using the compound method based on financial accounts. The case of the School of Forestry Engineering, Technical University of Madrid. Journal of Cleaner Production, v.66, p.224-232, 2014.

ASCUI, F.; LOVELL, H.. As frames collide: Making sense of carbon accounting. Accounting, Auditing and Accountability Journal, v.24, n.8, p.978-999, 2011.

CAREY, M.. Qualitative research skills for social work: Theory and practice. Ashgate Publishing Ltd., 2012.

CARO, D.; PULSELLI, F. M.; BENETTO, E.; RUGANI, B.. Implications of a consumer-based perspective for the estimation of GHG emissions. The illustrative case of Luxembourg. Science of the Total Environment, v.508, p.6775, 2015. DOI:

http://doi.org/10.1016/j.scitotenv.2014.11.053

CHOI, B. B.; LEE, D.; PSAROS, J.. An analysis of Australian company carbon emission disclosures. Pacific Accounting Review, 2013.

FONSECA, J. J. S.. Apostila de metodologia da pesquisa científica. 2002.

FERREIRA, N. S; YOSHIDA, E. M. P.. Produção científica sobre psicoterapias breves no Brasil e demais países latinoamericanos (1990-2000). Est. Psicol. Natal, v.3, n.3, p.523531, 2004.

GIL, A. C.. Como elaborar projetos de pesquisa. São Paulo: Atlas, 2002.

HRASKY, S.. Carbon footprints and legitimation strategies: symbolism or action?. Accounting, Auditing \& Accountability Journal, v.25, n.1, p.174-198, 2011.
JAMALUDIN, N. F.; AB MUIS, Z.; HASHIM, H.. An integrated carbon footprint accounting and sustainability index for palm oil mills. Journal of Cleaner Production, v.225, p.496509, 2019.

LIDDLE, B.. Consumption-based accounting and the tradecarbon emissions nexus. Energy Economics, v.69, p.71-78, 2018.

LOVELL, H.; MACKENZIE, D.. Accounting for carbon: the role of accounting professional organisations in governing climate change. Antipode, v.43, n.3, p.704-730, 2012.

MAALOUF, A.; EL-FADEL, M.. Towards improving emissions accounting methods in waste management: $A$ proposed framework. Journal of Cleaner Production, v.206, p.197210, 2019.

MELO, T.. Determinants of corporate social performance: the influence of organizational culture, management tenure and financial performance. Social Responsibility Journal, 2012.

RANKIN, M.; WINDSOR, C.; WAHYUNI, D.. An investigation of voluntary corporate greenhouse gas emissions reporting in a market governance system. Accounting, Auditing \& Accountability Journal, v.24, p.1037-1070, 2011. DOI: http://doi.org/10.1108/09513571111184751

RICHARDSON, R. J.. Pesquisa social: métodos e técnicas. 4 ed. São Paulo: Atlas, 2017.

SCHALTEGGER, S.; CSUTORA, M.. Carbon accounting for sustainability and management. Status quo and challenges. Journal of Cleaner Production, v.36, p.1-16, 2012.

STECHEMESSER, K.; GUENTHER, E.. Carbon accounting: A systematic literature review. Journal of Cleaner Production, v.36, p.17-38, 2012.

ZVEZDOV, D.; SCHALTEGGER, S.. Decision support through carbon management accounting-A framework-based literature review. Corporate Carbon and Climate Accounting, p.27-44, 2015.

A CBPC - Companhia Brasileira de Produção Científica (CNPJ: 11.221.422/0001-03) detém os direitos materiais desta publicação. Os direitos referem-se à publicação do trabalho em qualquer parte do mundo, incluindo os direitos às renovações, expansões e disseminações da contribuição, bem como outros direitos subsidiários. Todos os trabalhos publicados eletronicamente poderão posteriormente ser publicados em coletâneas impressas sob coordenação da Sustenere Publishing, da Companhia Brasileira de Produção Científica e seus parceiros autorizados. Os (as) autores (as) preservam os direitos autorais, mas não têm permissão para a publicação da contribuição em outro meio, impresso ou digital, em português ou em tradução. 